\title{
Conceptual Design of Weapon Equipment System-of- Systems Based on Capability
}

\author{
Hao Chen, Jian Huang*, Jianxing Gong and Jiangtao Kong \\ College of Artificial Intelligence, National University of Defense Technology, Changsha 410073, China \\ ${ }^{*}$ Corresponding author
}

\begin{abstract}
In response to the complex process and the lack of response to capability requirements of operational concept design, this paper proposes a conceptual design method for weapon equipment system-of-systems based on capability requirements. The operational concept design is divided into two parts: "operational capability requirements modeling" and "capabilitybased conceptual design modeling”. The corresponding modeling framework and description products are presented separately. Finally, using the method proposed in this paper, the conceptual design tool of weapon equipment system-of-systems is proposed. The superiority and practicability of the method and the tool are verified by an example.
\end{abstract}

Keywords-weapon equipment system-of-systems; operational concept; operational capability; conceptual design

\section{INTRODUCTION}

With the continuous development of science and technology and military technology, the future information warfare will no longer be confined to the independent operations of a single army or the joint operations of several armies and services, but will be an integrated joint operation that tends to break the boundaries of the the military. Based on actual combat requirements, it is an urgent problem to rapidly establish an integrated weapon equipment system-of-systems (WESoS) that meets the needs of capabilities, aiming at the ever-changing battlefield environment and complex missions.

Conceptual design is a continuous and in-depth process from macro to concrete, from coarse to fine-grained. It is a series of organized and purposeful activities for specific design goals [1]. If the WESoS is regarded as a special product, the process of transforming the mission requirements into its equipment requirements can be abstracted into the process of designing the concept of operation [2]. The US and other countries have already had a considerable application for operational concept design [3][4]. They are mostly used to clarify mission tasks, plan operational capabilities, and guide the construction of military systems and the use of combat forces.

Operational concept design belongs to the category of complex systems, which involves many fields, such as operations, command, equipment, and technology. System-ofsystems (SoS) architecture refers to the structure of the various components of systems, the relationships between them, and the principles and guidelines that govern their design and evolution over time [5]. Considering and describing the operational concept from the perspective of WESoS architecture is an effective way to achieve conceptual design [6]. At present, most of the researches on WESoS architecture are guided by the US Department of Defense Architecture Framework (DoDAF) [5][7]. Li Daxi et al. using DoDAF established the visual models of architecture and operation requirements for airborne anti-missile equipment [8]. Li Zhihuai et al. proposed an evaluation methodology based on capability viewpoint of DoDAF [9]. Fan Yanping et al., referring to the DoDAF specification, tried to propose the engineering-oriented development theory and method of WESoS requirements, provided a set of solutions including methods, models, tools and resources to improve the scientific and normative of the WESoS requirements development [10]. In order to study the semantic WESoS, Li Kang et al. introduced semantic meta-model based on DoDAF meta-model, then constructed ontology models of combat view, system view, and capability view [11]. Hu Jianpeng et al., referring to the part of DoDAF 2.0's viewpoints and SysML modeling language, discussed a generic modeling approach to SoS architecture based on parallel discrete event system specification [12].

In recent years, researches on operational concept design have also achieved some results. Hu Jianwen et al. proposed some principles and three key elements for concept modeling of WESoS, introduced function-capability hierarchical graph and entity structure hierarchical graph [13]. To overcome the deficiencies of the concept-based equipment development demonstration, a model-based equipment operational concept design method is presented [14]. In the way of model-based architecture and architecture-based design, this method established 19 DoDAF views based on SysML. In the view of DoDAF, Li Longyue et al. presented a military concept modeling method in UML, based on which, a method to design the ballistic missile defense system is given [15]. An agentoriented conceptual design method was introduced to guide the analysis and design of conceptual models for missile combat simulation, which makes it possible that some factors such as entity, activities, and interactions among the systems could be abstracted at the beginning of modeling [16].

Through the above analysis, it can be seen that many achievements have been obtained in the research on the conceptual design of the WESoS, but some problems still to be solved are also exposed:

- The purpose of constructing the WESoS architecture for operational concept design is to achieve certain capabilities, fulfill certain missions, and meet certain 
requirements under the complicated and varied battlefield environment conditions. In the existing research, the conceptual design process only focuses on the design of key capability requirements, or only takes the capability requirements as a description perspective, but does not put the operational capability throughout the process, which results in a lack of response to the capability requirements.

- At present, the design process of the WESoS architecture concept is complex and inefficient, and it is difficult to cope with the ever-changing battlefield situation. It is hard to quickly construct a corresponding WESoS plan according to the operational capability requirements. In many weapons and equipment, how to quickly find a matching weapon or equipment system according to the operational capability requirement is an urgent problem to be solved.

- Most of the existing conceptual design methods do not strictly define the content of products, the relationship between the product is not clear enough, and the consistency of the data is difficult to guarantee. The reusability of concept designs is low, which increases the development cost and difficulty.

- Most of the researches are only in the theoretical stage, and there is no complete integrated development environment, which makes the conceptual design inefficient and impractical.

To solve these deficiencies, this paper presents a conceptual design method of WESoS based on capability, which integrates the capability requirements into the entire conceptual design process. Starting from the operational mission, the method analyzes the operational capability requirements of the WESoS from the top down, transforming the abstract mission tasks into specific WESoS solutions through the operational capability requirements. This method establishes the relationship between capability and weaponry or equipment system, which improves the efficiency of the conceptual design. The operational concept design tool developed in this paper ensures data consistency and design reusability, which reduces development costs and difficulties.

\section{THE IDEA OF CONCEPTUAL DESIGN BASED ON CAPABILITY}

In order to achieve certain capabilities and satisfy certain missions under the complicated battlefield environment conditions, this paper presents a conceptual design method for WESoS based on capability requirements. The idea of conceptual design based on capability is shown in Figure 1.

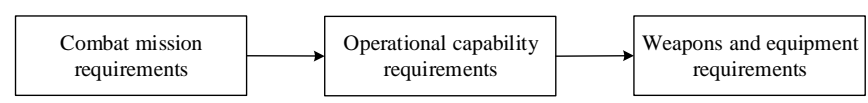

FIGURE I. REQUIREMENT TRANSFORMATION IN CONCEPTUAL DESIGN.

The essence is to transform the abstract mission requirements into specific weapon equipment requirements through capacity requirements. It can not only choose weapons and equipment that are suitable for combat missions from a large library of weapons and equipment. Moreover, starting from the demand for operational capability, it has a clear and direct guiding role for the determination of the operational capabilities, performance indicators and technical indicators of future weapons and equipment, and proposes constructive development ideas for the evolution of weapons and equipment.

The operational concept design process is divided into two parts: "operational capability requirements (OCR) modeling" and "capability-based conceptual design (CCD) modeling". CCD modeling is carried out on the basis of OCR modeling. Figure 2 demonstrates the relationships between the two modeling processes. The modeling of operational capability requirements is to map the operational mission to WESoS capability requirement. Then the WESoS capability is decomposed step by step and finally transformed into the equipment capability. The OCR modeling provides the CCD modeling with all levels of operational capability requirements.

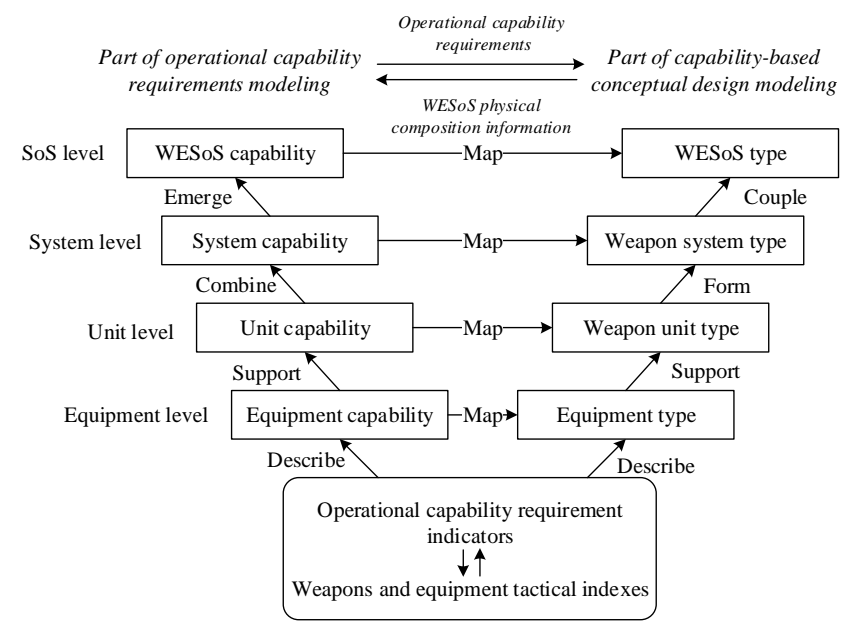

FIGURE II. THE RELATIONSHIPS BETWEEN THE TWO MODELING PROCESSES.

The CCD modeling maps the hierarchy of operational capability requirements determined in OCR modeling to WESoS physical composition relationships. On this basis, CCD modeling also needs to build engagement and interaction relationships to complete the operational concept design of WESoS, which is described in detail in Section III.

\section{CAPABILITY-BASED OPERATIONAL CONCEPT DESIGN FRAMEWORK}

\section{A. OCR Modeling Framework and Products}

In the capability-based operational concept design framework, OCR modeling is the basis and preconditions of CCD modeling, guiding the conceptual design process to be carried out step by step according to the operational capability requirements of each level.

OCR modeling is the process of transforming the operational mission into operational capabilities at all levels. The ultimate goal of modeling is to clarify the operational capability requirements at each level in Figure 2 and to form an 
operational capability requirement hierarchical relationship tree in form of "operational mission — WESoS capability system capability - unit capability - equipment capability”. The framework and products (OCR-1-OCR-6) of OCR modeling are illustrated in Figure 3.

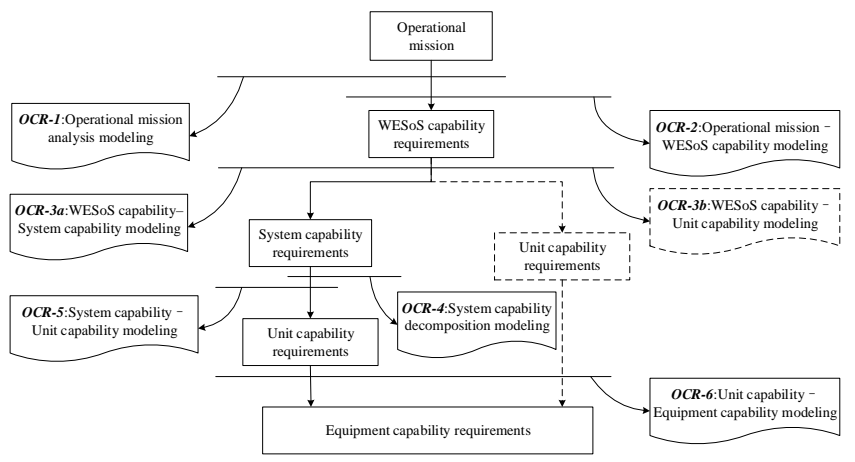

FIGURE III. OCR MODELING FRAMEWORK AND PRODUCTS.

OCR-1: Operational mission analysis modeling outlines the actual battlefield application background of the WESoS from the aspects of strategic objectives, combat mission, battlefield environment, enemy situation, etc. The battlefield environment describes the social and political environment in which the WESoS is located, as well as the natural environment such as meteorology and hydrology. The enemy situation specifically describes the current state and development trend of the enemy, including geographical location information, equipment composition information, staffing ratio information and development trends in the future. By doing this, targeted strategic deployment and strategic actions can be made more accurately. OCR-1 is presented by a description document.

OCR-2: Operational mission-WESoS capability modeling transforming the battlefield environment, mission tasks, and various social and political factors faced by the WESoS described in OCR-1 into the operational capability requirements at the SoS level. The WESoS capability requirements describe the types of operational capability and capability requirement indicators that the WESoS should have at the coarsest granularity. This modeling process demonstrates what SoS capabilities are needed to accomplish the operational mission. OCR-2 is presented in the form of a tree map or a tree mapping table.

OCR-3a(b): OCR-3 refines the WESoS capability into system capability requirements or unit capacity requirements which directly support WESoS capabilities. Due to the flexibility of the composition relationships and command relationships, the WESoS capability can be directly mapped to unit capacity requirements sometimes, which is illustrated by the dashed line in Figure 3. There is a many-to-many relationship between WESoS capabilities and system capabilities (unit capabilities), and an organic combination of different system capabilities (unit capabilities) emerges as new WESoS capabilities. OCR-3 can be implemented by a tree map or a mapping matrix.

OCR-4: System capability decomposition modeling further decomposes the system capability requirements obtained in OCR-3 until a system capability can be implemented in a single system, which further clarifies the system capability requirements and lays the foundation for the determination of unit capability requirements. OCR-4 is presented in a tree map.

OCR-5: System capabilities in the system capability-unit capability modeling refers to the lowest level of system capability in OCR-3a and OCR-4, i.e., the part that doesn't have subsystem capabilities. The unit here is the smallest unit module that constitutes WESoS. The various units that make up the same system capability may have similar capabilities, or the capabilities of various units have strong correlations to jointly achieve a certain system capability. OCR-5 is presented in the form of a tree map or a tree mapping matrix.

OCR-6: Unit capability-equipment capability modeling maps the unit capabilities identified by OCR-3b and OCR-5 to combat equipment entity capabilities. As shown in Figure 2, the operational capability requirements indicators and equipment tactical indexes establish the underlying relationships between OCR modeling and CCD modeling. The capability requirement of the equipment is expressed as the equipment tactical index requirement of the devices carried by the unit. In this way, the unit can achieve different capabilities by carrying different equipment. OCR-6 is established by a mapping diagram.

\section{B. CCD Modeling Framework and Products}

CCD modeling carries out conceptual design in multiple dimensions. Based on the hierarchical relationship tree of operational capability proposed in OCR products, CCD modeling completes the design of the physical composition, operational concept and interaction relationship of the WESoS.

The interaction relationship in operational concept design includes two categories: information interaction relationship and engagement interaction relationship. The role of information interaction relationship is to enable WESoS to implement its corresponding operational capability, ensure the normal operation of each unit, and realize interconnection, intercommunication, and interoperability between units. Information interaction relationships include command relationship, reporting relationship, collaborative relationship, etc. Engagement interaction relationship, which aims at enemy systems or units, is to complete the operational tasks and achieve the strategic goals. It includes reconnaissance relationship, positioning relationship, interference relationship, strike relationship, etc.

The framework and products (CCD-1-CCD-6) of CCD modeling are illustrated in Figure 4.

CCD-1: Operational capability-WESoS type modeling maps the operational capability requirement hierarchical relationship tree obtained in OCR modeling into the WESoS types. As shown in Figure 2, it should be noted that the WESoS type here is a broad concept, including the type of WESoS, the type of system, the type of unit and the type of equipment. Through CCD-1, a "WESoS type - system type - unit type - equipment type” WESoS requirements hierarchical relationship tree is formed, which lays a foundation for the determination of the WESoS. CCD-1 is presented in a mapping matrix. 


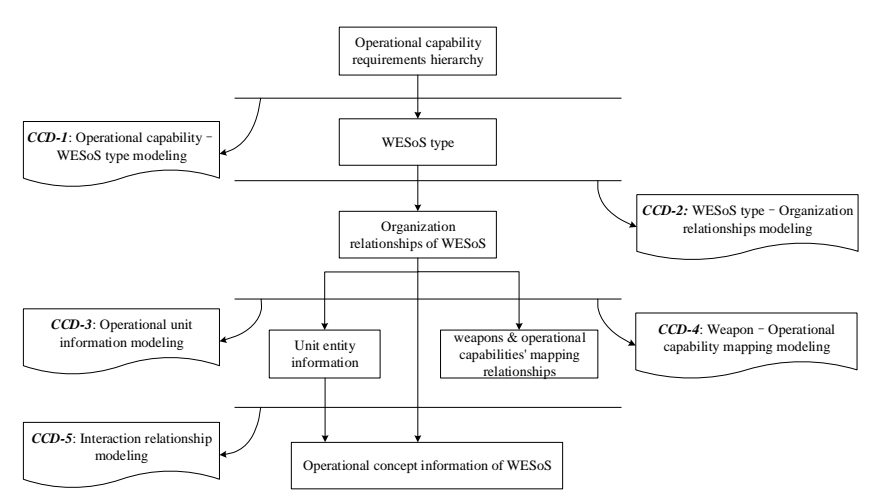

FIGURE IV. CCD MODELING FRAMEWORK AND PRODUCTS.

CCD-2: WESoS type-organization relationships modeling is to map the WESoS types into a specific WESoS, forming a tree structure of WESoS such as "operational mission operational WESoS - operational system - operational unit - operational equipment". It should be noted that the WESoS type here is also a broad concept. In practical application, the WESoS type can be mapped into an existing SoS, system, unit or equipment with corresponding capabilities, or it can be mapped into a non-existence, which guiding the construction of the new weapons and equipment. CCD-2 specifies the physical composition of the WESoS and can be presented in a tree map.

CCD-3: Operational unit information modeling is the modeling of units identified in CCD-2. It describes the basic attribute information, tactical indicators and related information of the equipment carried by the unit. CCD-3 lays a foundation for the subsequent validation and analysis of the operational concept and the optimization of WESoS. CCD-3 is implemented by an information table.

CCD-4: Weapon-operational capability mapping modeling establishes the mapping relationship between units, equipment in CCD-2 and operational capabilities in OCR-6. CCD-4 clarifies which units and equipment support a certain operational capability, and what capabilities a certain unit or equipment should possess to meet the operational mission requirements. It plays an important role in guiding the construction of WESoS architecture, discovering capacity gaps, and optimizing the WESoS architecture. CCD-4 is implemented by mapping matrix.

CCD-5: Interaction relationship modeling is based on the physical composition information in CCD-2 and the unit entities in CCD-3, modeling the information interaction relationship and engagement interaction relationship of the WESoS. It includes the start and end node, the information data stream, the format and the content of the information, the communication protocol, and so on. The resulting operational concept information is a strategic overview based on the operational mission and operational capability requirements, and it visually demonstrates strategic intent and key points in achieving objectives. CCD-5 is shown as a description diagram.

\section{OPERATIONAl CONCEPT DESIGN TOOL FrameWORK}

Capability-based operational concept design tool is an implementation of the methodology described in Section III. If the relationship between conceptual design and optimization is studied from the perspective of control theory, the core idea can be described as Figure 5. Our tool implements the part inside the dashed box.

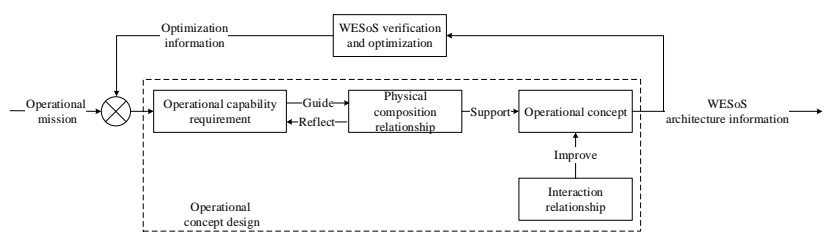

FIGURE V. THE IDEA OF WESOS CONCEPTUAL DESIGN, VERIFICATION AND OPTIMIZATION.

As shown in the dashed box, the entire conceptual design tool includes the description of operational capability requirements, physical composition relationships, interaction relationships, and operational concept. The operational capability requirements guide the construction of the physical composition of the WESoS. On the other hand, physical composition reflects the operational capabilities. At the same time, the physical composition of the WESoS is the basis for the description of the operational concept, and the configuration of the interaction relationship further improves the operational concept information. The implementation framework of the conceptual design tool is shown as Figure 6.

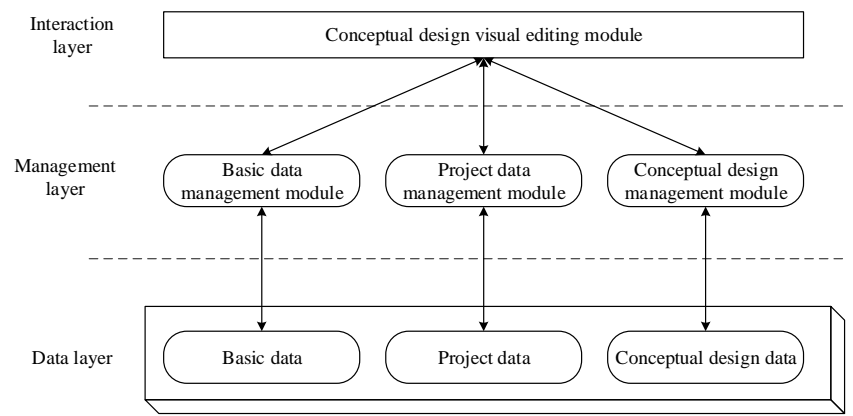

FIGURE VI. THE IMPLEMENTATION FRAMEWORK OF THE CONCEPTUAL DESIGN TOOL.

There are three layers constructs the framework, from top to bottom, the interaction layer, the management layer, and the data layer. The interaction layer provides a visual editing operation interface for each module function realization in the management layer. Through the interaction layer, users can not only manage the basic data, but also complete the conceptual design of the WESoS. The data layer is the foundation and plays an important role in the tool. The basic data includes enemy and our weapons and equipment data, combat engineering data, combat material data and battlefield environmental data. It not only contains basic attribute information of the entity, such as name, picture, military symbol, etc. but also information about its tactical indicators. The basic data is the data foundation for conceptual design. The project data describes the details of the project itself, including basic information such as project name, creation time, and creation purpose. The conceptual design data corresponds to the project data, including all data generated during OCR and CCD modelings, such as operational capability decomposition mapping information, physical composition information, and operational concept description information. 


\section{InSTANCE APPLICATION OF THE TOOL}

In this section, we will present the operational concept design tool through an example application. Basic data management tool is the foundation of conceptual design and will be introduced first.

\section{A. Basic Data Management Tool}

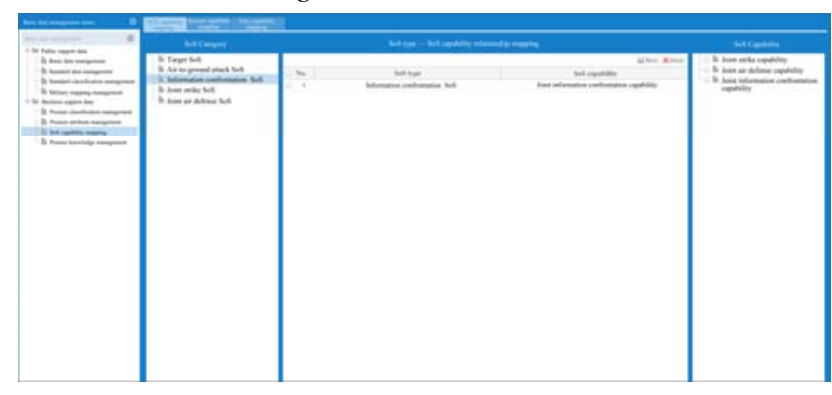

FIGURE VII. THE PARTIAL SCREEN SHOT OF THE BASIC DATA MANAGEMENT TOOL.

Figure 7 is a partial screen shot of the basic data management tool. It not only realizes the addition, deletion, and editing of the basic data, but also can configure interactive information. Through the associated operation of the basic data, the combat capability is mapped to the SoS type, system type, unit type, and equipment type. Figure 7 is the process of mapping the SoS operational capability to the WESoS type. The mapping table in the figure reflects the modeling information in the modeling description product CCD-1. In addition, the mapping relationship between weapon type and specific unit and equipment is also implemented in the basic data management tool, so the tool also contains some information in CCD-2.

\section{B. Application of the Conceptual Design Tool}

This section applies the conceptual design tool to the operational conceptual design of a case and explains the correctness of the method and the superiority of the tool. This tool has three main viewpoints, namely, the operational capability viewpoint, the physical composition viewpoint, and the operational concept viewpoint, which will be described in detail in the following case.

The basic idea of the case is: in order to safeguard the reunification of the motherland and ensure the territorial integrity, the red and blue sides conducted military exercises in a certain area. The red side received orders from its superiors to quickly organize its troops and attack the blue ground station, the reporting center, and the command center.

Take the red side as an example. The first step is to conduct operational mission analysis modeling, clarify strategic objectives, application background of the WESoS and the battlefield environment, which corresponds to the description product OCR-1. In the conceptual design tool, OCR-1 is presented by a description document, as shown in Figure 8.

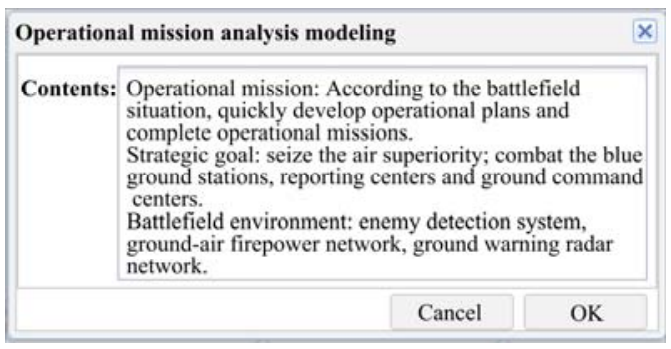

FIGURE VIII. OCR-1 IN THE TOOL.

The OCR modeling process is mainly reflected in the operational capability viewpoint, as shown in Figure 9 (partial screenshot of the tool). In the figure, an operational capability requirement hierarchical relationship tree is formed. The operational capability data in the figure comes from the basic data, which is configured by the user. The products corresponding to OCR modeling is marked on the figure.

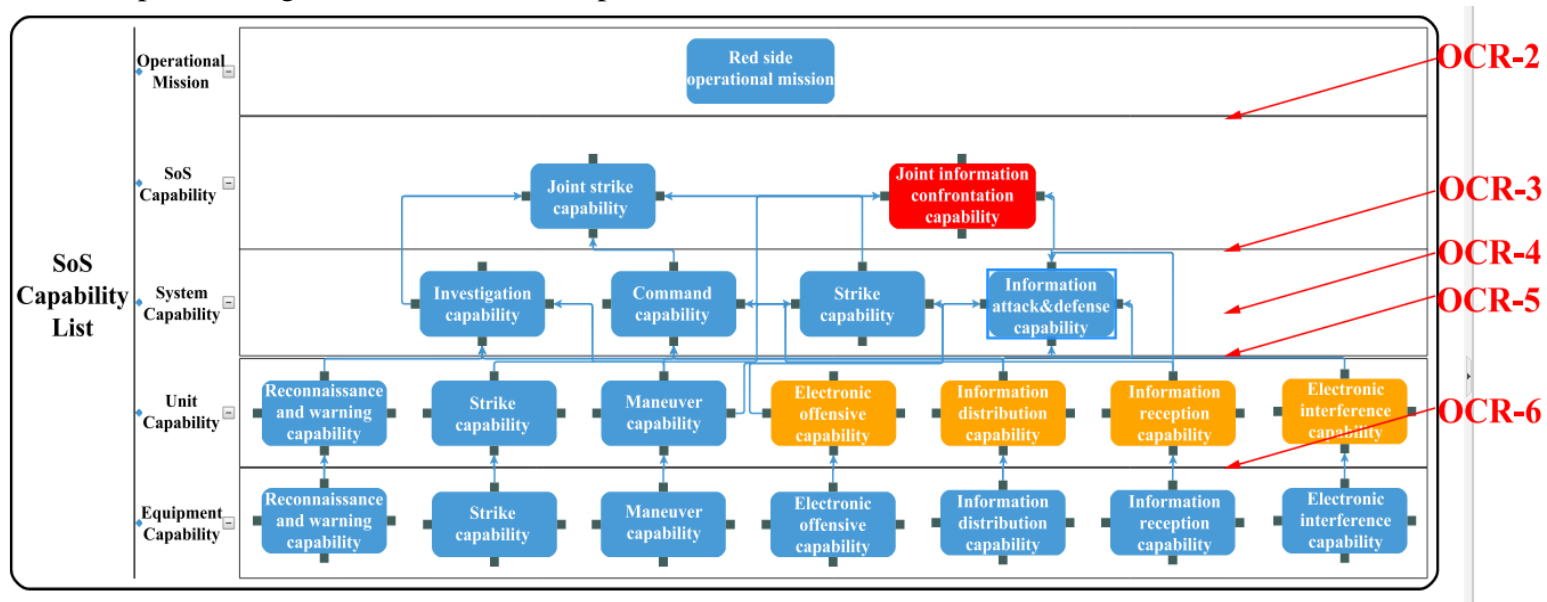

FIGURE IX. OPERATIONAL CAPABILITY VIEWPOINT.

Physical composition viewpoint illustrates the modeling process from capability requirements to physical composition information of the corresponding WESoS. The physical composition viewpoint related to the case is shown in Figure 10 (partial screenshot of the tool). According to the mapping relationships between the predefined weapon equipment SoS (system, unit, equipment) type and the corresponding operational capability in the basic data management tool, when 
a specific system is selected, the conceptual design tool quickly filters out the units and equipment that meet the corresponding capability requirement for the user to select. Reflecting CCD-4, the automatically generated capability mapping matrix is shown in Figure 11 (partial screenshot of the tool). The corresponding product in the third section is marked in the figure.

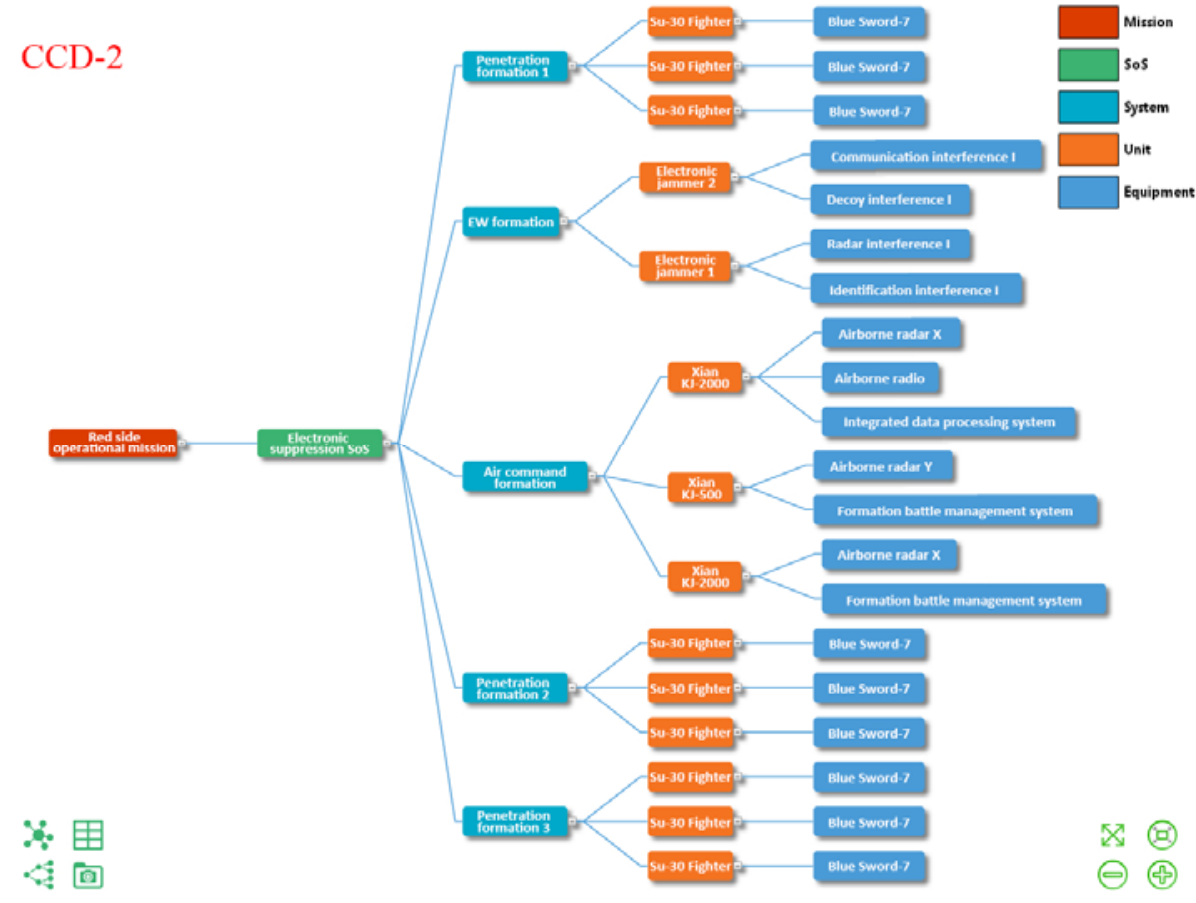

FIGURE X. PHYSICAL COMPOSITION VIEWPOINT.

\begin{tabular}{|c|c|c|c|c|c|c|c|c|}
\hline \multicolumn{9}{|c|}{ Red side capability mapping matrix } \\
\hline Unit & Equipment & $\begin{array}{l}\text { Reconnaissance } \\
\text { and warning } \\
\text { capability }\end{array}$ & $\begin{array}{c}\text { Strike } \\
\text { capability }\end{array}$ & $\begin{array}{l}\text { Maneuver } \\
\text { capability }\end{array}$ & $\begin{array}{l}\text { Electronic } \\
\text { offensive } \\
\text { capability }\end{array}$ & $\begin{array}{l}\text { Information } \\
\text { distribution } \\
\text { capability }\end{array}$ & $\begin{array}{c}\text { Information } \\
\text { reception } \\
\text { capability }\end{array}$ & $\begin{array}{c}\text { Electronic } \\
\text { interference } \\
\text { capability }\end{array}$ \\
\hline Su-30 Fighter & Blue Sword-7 & $x$ & $\checkmark$ & $\checkmark$ & $x$ & $x$ & $x$ & $x$ \\
\hline Su-30 Fighter & Blue Sword-7 & $x$ & $\checkmark$ & $\checkmark$ & $x$ & $x$ & $x$ & $x$ \\
\hline Su-30 Fighter & Blue Sword-7 & $x$ & $\checkmark$ & $\checkmark$ & $\times$ & $x$ & $x$ & $x$ \\
\hline \multirow{2}{*}{$\begin{array}{l}\text { Electronic } \\
\text { jammer 2 }\end{array}$} & $\begin{array}{c}\text { Communication } \\
\text { interference } 1\end{array}$ & $\checkmark$ & $\times$ & $\times$ & $\checkmark$ & $\checkmark$ & $\checkmark$ & $\checkmark$ \\
\hline & Decoy interference I & $\checkmark$ & $\times$ & $\times$ & $\checkmark$ & $\checkmark$ & $\checkmark$ & $\checkmark$ \\
\hline \multirow{2}{*}{$\begin{array}{l}\text { Electronic } \\
\text { jammer } 1\end{array}$} & Radar interference I & $\checkmark$ & $\times$ & $x$ & $\checkmark$ & $\checkmark$ & $\checkmark$ & $\checkmark$ \\
\hline & $\begin{array}{l}\text { Identification } \\
\text { interference I }\end{array}$ & $\checkmark$ & $x$ & $x$ & $\checkmark$ & $\checkmark$ & $\checkmark$ & $\checkmark$ \\
\hline \multirow{3}{*}{ Xian KJ-2000 } & Airborne radar $X$ & $\checkmark$ & $\times$ & $\times$ & $\checkmark$ & $\checkmark$ & $\checkmark$ & $\checkmark$ \\
\hline & Airborne radio & $\checkmark$ & $\times$ & $\times$ & $\checkmark$ & $\checkmark$ & $\checkmark$ & $\checkmark$ \\
\hline & $\begin{array}{l}\text { Integrated data } \\
\text { processing system }\end{array}$ & $\checkmark$ & $x$ & $x$ & $x$ & $\checkmark$ & $\checkmark$ & $\checkmark$ \\
\hline \multirow[b]{2}{*}{ Xian KJ-500 } & Airborne radar $Y$ & $\checkmark$ & $x$ & $x$ & $\checkmark$ & $\checkmark$ & $\checkmark$ & $\checkmark$ \\
\hline & $\begin{array}{l}\text { Formation battle } \\
\text { management system }\end{array}$ & $\checkmark$ & $\times$ & $\times$ & $\times$ & $\checkmark$ & $\checkmark$ & $\checkmark$ \\
\hline \multirow[b]{2}{*}{ Xian KJ-2000 } & Airborne radar $X$ & $\checkmark$ & $x$ & $x$ & $\checkmark$ & $\checkmark$ & $\checkmark$ & $\checkmark$ \\
\hline & $\begin{array}{c}\text { Formation battle } \\
\text { management system }\end{array}$ & $\checkmark$ & $x$ & $\times$ & $\times$ & $\checkmark$ & $\checkmark$ & $\checkmark$ \\
\hline Su-30 Fighter & Blue Sword-7 & $x$ & $\checkmark$ & $\checkmark$ & $x$ & $x$ & $x$ & $x$ \\
\hline Su-30 Fighter & Blue Sword-7 & $\times$ & $\checkmark$ & $\checkmark$ & $\times$ & $\times$ & $\times$ & $x$ \\
\hline Su-30 Fighter & Blue Sword-7 & $\times$ & $\checkmark$ & $\checkmark$ & $\times$ & $\times$ & $x$ & $\times$ \\
\hline Su-30 Fighter & Blue Sword-7 & $x$ & $\checkmark$ & $\checkmark$ & $\times$ & $x$ & $x$ & $x$ \\
\hline Su-30 Fighter & Blue Sword-7 & $\times$ & $\checkmark$ & $\checkmark$ & $x$ & $\times$ & $x$ & $x$ \\
\hline Su-30 Fighter & Blue Sword-7 & $\mathrm{x}$ & $\checkmark$ & $\checkmark$ & $x$ & $x$ & $x$ & $x$ \\
\hline
\end{tabular}

FIGURE XI. CAPABILITY MAPPING MATRIX CCD-4.

The contents of the operational concept viewpoint are mainly related to the modeling description product CCD-5, as shown in Figure 12 (partial screenshot of the tool). Based on the operational capability viewpoint and the physical composition viewpoint, it is a strategic overview formed by combining operational missions and strategic objectives, by 
configuring information interactions and engagement interactions. The list of elements in the left panel shows all the elements in the physical composition viewpoint of both red and blue in a hierarchical diagram. The right panel provides a list of relationships for storing information interactions and engagement interactions. Each interaction relationship corresponds to different attribute information and parameter indicators in the database.

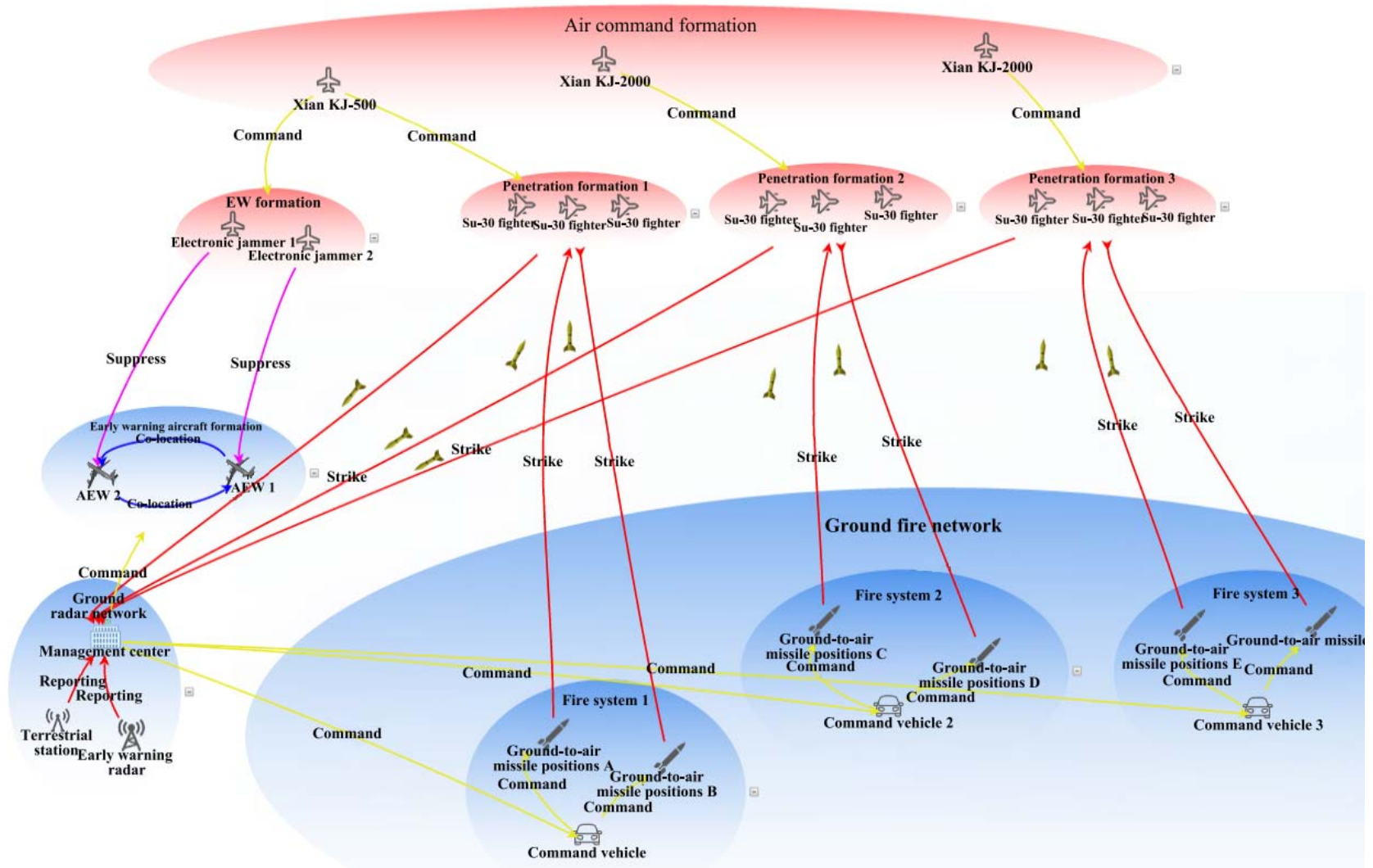

FIGURE XII. OPERATIONAL CONCEPT VIEWPOINT.

So far, the modeling and description products introduced in section III are implemented in the conceptual design tool. From the perspective of the entire design process, the idea of capacity requirement runs through, and the tool proposed in this paper does not mechanically stack all the modeling description products together but integrate the products into the whole conceptual design process. The process of conceptual design is the process of generating and storing the corresponding description product. The whole concept design process is interlocking, reflecting the idea of structured modeling methods and ensuring the consistency and completeness of data. Each individual project can be used as a template in subsequent construction, which improves development efficiency and ensures the standardization, rationality, and scientificity of the entire conceptual design process.

\section{CONCLUSION}

This paper presents a conceptual design method of WESoS based on capability, which integrates the capability requirements into the entire conceptual design process. Starting from the operational mission, the method analyzes the operational capability requirements of the WESoS from the top down, transforming the abstract mission tasks into specific WESoS solutions. This method establishes the relationship between capability and weaponry or equipment system, which improves the efficiency of the conceptual design. The operational concept design tool developed in this paper provides an integrated development environment, which ensures data consistency and reusability and reduces the development costs and difficulties.

\section{REFERENCES}

[1] Hsu W, Liu B. Conceptual design: issues and challenges[J]. ComputerAided Design, 2000, 32(14):849-850.

[2] Qiaoli L I, Guo Q, Yang X. A New Angle of View About the Analysis of Weapon Equipment Requirements[J]. Journal of the Academy of Equipment Command \& Technology, 2009.

[3] Documents G. Quadrennial Defense Review Report (February 2010)[M]. IEEE USA Books \& eBooks, 2011.

[4] Australian Defence Force. Future maritime operating concept 2025, maritime force projection and control, 2007.

[5] CIO DoD. Dod architecture framework version 2.02. DoD Deputy Chief Information Officer, Available online at http://dodcio. defense. gov/dodaf20/dodaf20 pes. aspx, accessed Nov, 2012.

[6] Guang Lin Ma, Xi Quan Xie, and Ming Jie Gao. New equipment combat concept design framework. Military Operations Research and Systems Engineering, (1):5-13, 62012.

[7] DoD Architecture Framework Working Group et al. Dod architecture framework version 1.0. Department of Defense, 2003. 
[8] Li D, Zhang Q, Li X, et al. Architecture modeling for equipment of airborne anti-missile based on DoDAF[J]. XI Tong Gong Cheng Yu Dian Zi Ji Shu/systems Engineering \& Electronics, 2017, 39(5):10361041.

[9] L. I. Zhihuai, Xiansi Tan, Hong Wang, and Yanjun Yao. The evaluation method of weapon equipment system-of-systems based on capability viewpoint of dodaf. Journal of Academy of Equipment, 2012.

[10] Yan Ping Fan, Qi Sheng Guo, M. U. Ge, and Jing Hua Song. Complexity of weapon system of systems requirement development and its solution. Systems Engineering \& Electronics, 36(7):1320-1327, 2014.

[11] L. I. Kang, L. I. Xin-Ming, and Liu Dong. Modeling method for weapon system of systems structure based on semantic meta-model. Systems Engineering \& Electronics, 2015.

[12] Jianpeng $\mathrm{Hu}$ and Linpeng Huang. Modeling and simulation of executable architecture based on p-devs. Journal of System Simulation, 2016.

[13] H. U. Jian-Wen, H. U. Xiao-Feng, and Li Li Zhu. Concept modeling and verifying analysis for weapon and armament system of systems. Journal of System Simulation, 18(12):3630-3633, 2006.

[14] Ming Hu Tian, Yan Ping Fan, and Qi Sheng Guo. Model-based equipment operational concept design method. Journal of Academy of Armored Force Engineering, 2015.

[15] Long-Yue L I, Liu F X. Concept Model and Simulation System Design of the Ballistic Missile Defense Based on DoDAF Views[J]. Command Control \& Simulation, 2012.

[16] L. I. Ying and B. I. Yi-Ming. Design of agent-oriented conceptual model for missile combat simulation. Systems Engineering \& Electronics, 32(1):90-93,2010.s 